\title{
Progression of diabetic retinopathy during pregnancy in women with type 2 diabetes
}

\author{
K. L. Rasmussen • C. S. Laugesen • L. Ringholm • \\ M. Vestgaard • P. Damm • E. R. Mathiesen
}

Received: 1 November 2009 / Accepted: 22 January 2010/Published online: 12 March 2010

(C) Springer-Verlag 2010

\begin{abstract}
Aims/hypothesis We studied the progression of diabetic retinopathy during pregnancy in women with type 2 diabetes.

Methods Fundus photography was performed at median 10 (range 6-21) and 28 (27-37) gestational weeks in 80 of 110 (73\%) consecutively referred pregnant women with type 2 diabetes. Diabetic retinopathy was classified in five stages.
\end{abstract}

K. L. Rasmussen $(\bowtie) \cdot$ L. Ringholm $\cdot$ M. Vestgaard •

E. R. Mathiesen

Department of Endocrinology, Rigshospitalet,

Copenhagen University Hospital,

Blegdamsvej 9,

2100 København Ø, Denmark

e-mail: katrinespostkasse@gmail.com

K. L. Rasmussen • L. Ringholm • M. Vestgaard • P. Damm •

E. R. Mathiesen

Center for Pregnant Women with Diabetes, Rigshospitalet,

Copenhagen University Hospital,

Copenhagen, Denmark

\section{P. Damm}

Department of Obstetrics, Rigshospitalet,

Copenhagen University Hospital,

Copenhagen, Denmark

\section{S. Laugesen}

Department of Ophthalmology, Rigshospitalet,

Copenhagen University Hospital,

Copenhagen, Denmark

\section{P. Damm • E. R. Mathiesen}

Faculty of Health Sciences, University of Copenhagen,

Copenhagen, Denmark

\section{S. Laugesen}

Department of Ophthalmology, Roskilde,

Copenhagen University Hospital,

Roskilde, Denmark
Progression was defined as at least one stage of deterioration of diabetic retinopathy and/or development of macular oedema on at least one eye between the two examinations. Macular oedema was defined as retinal thickening and/or hard exudates within a diameter of $1,500 \mu \mathrm{m}$ in the macula area.

Results Diabetic retinopathy, mainly mild, was present in $11(14 \%)$ women in early pregnancy. Median duration of diabetes was 3 years (range 0-16 years). At baseline, $\mathrm{HbA}_{1 \mathrm{c}}$ was $6.4 \%$ (1.0) (mean [SD]), systolic BP 121 (13) and diastolic BP 72 (9) $\mathrm{mmHg}$. Prior to pregnancy, 22 (28\%) women had been on insulin treatment. During pregnancy 74 women $(93 \%)$ were treated with insulin and $11(14 \%)$ with antihypertensive medication. Progression of diabetic retinopathy was observed in 11 (14\%) women. Progression was mainly mild, but one woman with poor glycaemic control and uncontrolled hypertension progressed from mild retinopathy to sight-threatening retinopathy with proliferations, clinically significant macular oedema and impaired vision in both eyes. Progression of diabetic retinopathy was associated with a longer duration of diabetes $(p=0.03)$ and insulin treatment before pregnancy $(p=0.004)$.

Conclusions/interpretation Despite a low risk of progression of retinopathy in pregnant women with type 2 diabetes, sight-threatening deterioration did occur.

Keywords Antihypertensive therapy - Diabetic retinopathy · Pregnancy · Type 2 diabetes
Abbreviations
CSMO Clinically significant macular oedema
SMPG Self-monitored plasma glucose
UAE Urine albumin excretion 


\section{Introduction}

Pregnancy-induced deterioration of diabetic retinopathy is well described in type 1 diabetes [1]. The risk of progression of diabetic retinopathy during pregnancy is generally regarded to be similar for type 1 and type 2 diabetic patients, but data supporting this assumption are few. The prevalence and progression of diabetic retinopathy in pregnancy complicated by type 2 diabetes have hardly been investigated hitherto [2-4].

The number of pregnant women suffering from type 2 diabetes is increasing $[3,5,6]$. In urban Northern European areas up to $50 \%$ of the pregnant women with pregestational diabetes currently have type 2 diabetes [3], making concern at the risk of pregnancy-induced progression of diabetic retinopathy in these patients highly relevant.

Preliminary results from our own centre suggest that less attention is focused on the risk of pregnancy-induced progression of diabetic retinopathy in women with type 2 diabetes than in those with type 1 diabetes [4]. Despite diabetic retinopathy prevalence of $18 \%$, only $60 \%$ of pregnant women with type 2 diabetes had the two recommended eye examinations performed in pregnancy [4].

In a large Japanese study, the prevalence of diabetic retinopathy in pregnant women with type 2 diabetes was $28 \%$, with $2 \%$ receiving laser treatment during pregnancy [2]. In another study, diabetic retinopathy in pregnant women with type 2 diabetes was described briefly, i.e. retinal status was determined at least once in more than $80 \%$ of the women [3].

Pregnant women with type 2 diabetes often have a relatively short known duration of diabetes and relatively good glycaemic control as measured by $\mathrm{HbA}_{1 \mathrm{c}}$ at first pregnancy visit $[3,5]$. This might be associated with a low risk of progression of diabetic retinopathy during pregnancy. However, pregnant women with type 2 diabetes often come from deprived areas where the risk of several complications to pregnancy is increased [3]. In addition regular attendance at a diabetes clinic, pregnancy planning and diabetic retinopathy screening are less frequent, which may lead to a more pronounced risk of progression of diabetic retinopathy.

The aim of this study was to document the prevalence and progression of diabetic retinopathy in an unselected population of pregnant women with type 2 diabetes using a standardised photoscreening technique.

\section{Methods}

We studied all singleton pregnancies from July 2003 to September 2008 in women with type 2 diabetes who had been referred to Rigshospitalet before 20 gestational weeks and delivered after 22 gestational weeks. The area of referral was Eastern Denmark and the Faroe Islands, with a population of approximately 2.50 million. If a woman delivered more than once in the study period, only the last pregnancy was included, leaving 110 eligible participants. Women whose medical record could not be identified $(n=2)$ or in whom less than two ophthalmological examinations were performed during pregnancy $(n=28)$ were excluded, leaving 80 women for the present study. The study was carried out in accordance with the Declaration of Helsinki (2000).

Ophthalmological examinations were performed on dilated pupils by photoscreening. Photos were taken by a specially trained nurse, as a part of our routine practice, twice in pregnancy, i.e. at the first pregnancy visit at a median of 10 (range 6-21) and at 28 (27-37) weeks. After dilatation a minimum of three fundus photographs of each eye were taken, including one macular-field with the optic disc positioned nasal in the horizontal plane, one nasal peripheral with the optic disc positioned temporal on the horizontal plane and one temporal with the macula positioned nasal on the horizontal plane. We used a highresolution digital camera (FF450 plus IR; Carl Zeiss Meditec, Jena, Germany) at an angle of 50 degrees. If the specially trained nurse who took the pictures detected more than mild non-proliferative retinopathy, another four pictures covering the periphery were taken. The camerabackpart was an H5 (Phase One, Frederiksberg, Denmark) with a spatial resolution of $2,256 \times 2,023$ corresponding to $5 \times 10^{6}$ pixels. In a few cases photoscreening was not performed, and an experienced ophthalmologist performed the eye examinations.

Diabetic retinopathy was classified in five stages (Table 1) based on the classification of Wilkinson et al. [7]. Grading of pictures and possible additional eye examinations were performed by the same trained ophthalmologist (C. S. Laugesen), who has extensive experience in the examination of diabetic retinopathy in pregnant women and a long-lasting close collaboration with our centre. Diabetic macular oedema was defined as retinal thickening and/or hard exudates within a diameter of $1,500 \mu \mathrm{m}$ from the centre of the macula. Presence of diabetic macular oedema was graded separately and classified as clinically significant macular oedema (CSMO) if significant changes were present within $500 \mu \mathrm{m}$ from the macular centre, foveola [8] or, in the remaining cases, mild to moderate diabetic macular oedema (Table 1). Presence of CSMO was verified by slit lamp biomicroscopy in all cases. The diagnosis of mild to moderate macular oedema was based on photoscreening alone.

Vision was assessed by Snellen's chart as best corrected visual acuity with stenopedic opening. Visual acuity of $6 / 6$ corresponds to 1.0. Loss of visual acuity was defined as a decrease of $\geq 0.2$ of visual acuity during pregnancy on at least one eye. 
Table 1 Five-stage disease severity classification for diabetic retinopathy and definition of diabetic macular oedema

\begin{tabular}{ll}
\hline Stage & Definition \\
\hline $\begin{array}{l}\text { Five-stage disease severity classification for } \\
\text { diabetic retinopathy }\end{array}$ & \\
$\begin{array}{l}\text { No diabetic retinopathy } \\
\text { Mild non-proliferative diabetic retinopathy }\end{array}$ & $\begin{array}{l}\text { None of the listed criteria below } \\
\text { Moderate non-proliferative diabetic }\end{array}$ \\
$\begin{array}{l}\geq 10 \text { punctiform intraretinal haemorrhages and/or microaneurysms in a quadrant of retina } \\
\text { retinopathy }\end{array}$ & $\begin{array}{l}\text { and/or a varying number of hard exudates and/or } 1-10 \text { cotton wool spots } \\
\text { Pre-proliferative diabetic retinopathy }\end{array}$ \\
$\begin{array}{l}\text { and/or intraretinal microvascular angiopathies (IRMA) and/or venous beading } \\
\text { Proliferative diabetic retinopathy }\end{array}$ & Neovascularisation and/or larger preretinal/vitreous haemorrhage \\
$\begin{array}{l}\text { Definition of diabetic macular oedema } \\
\text { No macular oedema }\end{array}$ & None of the listed criteria below \\
Mild-to-moderate macula oedema & Thickening of the retina and/or hard exudates within $500-1,500 \mu \mathrm{m}$ of the centre of the macula \\
CSMO & Thickening of the retina and/or hard exudates within $500 \mu \mathrm{m}$ of the centre of the macula \\
\hline
\end{tabular}

Progression was defined as at least one stage of deterioration of diabetic retinopathy and/or development of diabetic macular oedema on at least one eye. Progression from no retinopathy to mild retinopathy was also regarded as progression of diabetic retinopathy. Sight-threatening progression was defined as loss of visual acuity and/or need of laser treatment during pregnancy due to proliferative changes or presence of CSMO. The eye with the worst stage of deterioration in diabetic retinopathy determined the stage of diabetic retinopathy in each woman. Eye examinations were performed more frequently on the basis of individual judgment, mainly with repeated photoscreening within 4 to 6 weeks, if progression of at least two stages of retinopathy and presence of preproliferative or proliferative diabetic retinopathy or CSMO were found. Poor metabolic control or elevated blood pressure also led to more frequent photoscreening. Laser therapy was performed if new proliferations occurred. In the event of sight-threatening diabetic retinopathy, postpartum follow-up was performed with evaluation of visual acuity to determine whether the visual loss was temporary or permanent. Photoscreening was performed in 151 (94\%) of the 160 ophthalmological examinations. The remaining nine examinations were performed for practical reasons by slit lamp biomicroscopy and were mainly in women without diabetic retinopathy. Of these nine, four examinations were at first pregnancy visits and five at 28 weeks. The 11 women with progression of diabetic retinopathy during pregnancy were all photoscreened at first pregnancy visit and at 28 weeks. Double grading of the same pictures by two independent ophthalmologists was not performed.

The diagnosis of diabetes was based on the referring physician's examinations, mainly two fasting plasma glucose values exceeding $7 \mathrm{mmol} / \mathrm{l}$. Women with type 1 diabetes or cystic fibrosis were excluded and the remaining women were classified as type 2 diabetes.
The women were followed as outpatients every 1 to 2 weeks, mainly at our centre, unless the patient lived a long distance away, in which case some of the diabetes visits were performed at a local diabetes clinic. Diabetes treatment was diet, either alone or combined with insulin. The majority of the women were being treated with basalbolus insulin regimen (actrapid and insulatard), but 23 (31\%) of the insulin-treated women were on a mixture of fast- and intermediate-acting insulin twice daily (mixtard) throughout pregnancy.

The women were instructed to register their selfmonitored plasma glucose (SMPG) readings four to seven times daily in diabetes diaries and to adjust their insulin dose to maintain a preprandial SMPG in the range of 4.0 to $6.0 \mathrm{mmol} / \mathrm{l}$, a $90 \mathrm{~min}$ postprandial SMPG of 4.0 to $8.0 \mathrm{mmol} / \mathrm{l}$ and a pre-bedtime SMPG of 6.0 to $8.0 \mathrm{mmol} / \mathrm{l}$. The aim was to achieve $\mathrm{HbA}_{1 \mathrm{c}}$ below $6.0 \%$ in late pregnancy. $\mathrm{HbA}_{1 \mathrm{c}}$ was assayed by a latex immunoagglutination inhibition method using only the same analyser (DCA 2000; Bayer, Newbury, UK). Normal $\mathrm{HbA}_{1 \mathrm{c}}$ ranges were $4.7 \%$ to $6.3 \%$ outside pregnancy, $4.5 \%$ to $5.7 \%$ in early pregnancy and $4.4 \%$ to $5.6 \%$ in late pregnancy [9].

Blood pressure was measured with a digital blood pressure monitor (A\&D Instruments, Abingdon, UK) in a sitting position after 5 to $10 \mathrm{~min}$ of rest. Guidelines for initiation of antihypertensive therapy during pregnancy were blood pressure $\geq 135 / 85 \mathrm{mmHg}$ and/or urine albumin excretion (UAE) $\geq 300 \mathrm{mg} / 24 \mathrm{~h}$. Methyldopa was the firstchoice antihypertensive agent, with labetalol and/or nifedipine being added when indicated [10].

UAE was analysed by an enzyme-linked immunosorbent assay [11]. When 24-h urine samples were not available, albumin/creatinine mass ratio was performed in a random urine sample [10]. Elevated albumin excretion was defined as $\mathrm{UAE}>30 \mathrm{mg} / 24 \mathrm{~h}$. 
Estimation of gestational age was based on early ultrasound scanning. Preterm delivery was defined as delivery before 37 weeks.

Pre-eclampsia was defined as blood pressure above 140/ $90 \mathrm{mmHg}$ and proteinuria $(\geq 1+)$ on a sterile urinary dipstick or proteinuria of $>300 \mathrm{mg} / 24 \mathrm{~h}$ (proteinuria of $300 \mathrm{mg} / 24 \mathrm{~h}$ approximates to UAE of $190 \mathrm{mg} / 24 \mathrm{~h}$ ) after 20 gestational weeks [10].

Data are given as mean (SD), median (range) or numbers (\%). Statistical analyses were performed using unpaired $t$ test as well as Fisher's exact test as appropriate. Women with regression of diabetic retinopathy were included in the group with no progression. Differences were considered to be statistically significant at a two-sided $p$ value of $p<0.05$.

\section{Results}

Diabetic retinopathy was present in 11 (14\%) women in early pregnancy and progressed during pregnancy in 11 (14\%; Table 2). The cases of retinopathy progression were mainly mild (Tables 2 and 3), with new-onset of diabetic retinopathy occurring in eight women. However, one woman developed sight-threatening diabetic retinopathy in both eyes (Table 3).

At first pregnancy visit the diabetic women were generally well controlled with $\mathrm{HbA}_{1 \mathrm{c}}$ of $6.4 \%(1.0 \%)$ (mean [SD]) and blood pressure of 121 (13)/72 (9) $\mathrm{mmHg}$. Median duration of diabetes was 3 (range 0-16) years.

Of the eleven women with progression, seven (64\%) had been on insulin before pregnancy compared with 15 of the $69(22 \%)$ women without progression $(p=0.004)$. The majority of the women not treated with insulin at referral initiated insulin treatment within few weeks. Only six $(8 \%)$ women remained on diet alone during the entire pregnancy.

Women with progression of diabetic retinopathy during pregnancy were characterised by longer duration of diabetes $(p=0.03)$, higher prevalence of insulin treatment prior to pregnancy $(p=0.004)$ and a trend towards higher $\mathrm{HbA}_{1 \mathrm{c}}$ at baseline $(p=0.07)$ compared with women without progression (Table 2).

During pregnancy glycaemic control improved in patients with and without progression of diabetic retinopathy, leading to a similar level of $\mathrm{HbA}_{1 \mathrm{c}}$ close to the goal of treatment from 20 weeks and in the remaining part of pregnancy (Table 4). The decrease in $\mathrm{HbA}_{1 \mathrm{c}}$ from baseline to 34 weeks was significantly larger in women with progression than in those with no progression of diabetic retinopathy $(1.5 \%$ [1.1] vs $0.7 \%$ [1.0], $p=0.04)$.

Among the women without progression of diabetic retinopathy, five had been well controlled on antihypertensive treatment, mainly ACE inhibitors and labetalol, prior to pregnancy (Table 5), changing to methyldopa in early pregnancy or continuing on labetalol. Another five women
Table 2 Baseline clinical characteristics of 80 pregnant women with type 2 diabetes in relation to progression of diabetic retinopathy

Values are mean (SD) unless otherwise stated, using data from first pregnancy visit at 10 (6-21) weeks, unless indicated otherwise

${ }^{\text {a }}$ For insulin (insulin, oral + insulin) compared with no insulin (oral, diet) before pregnancy; ${ }^{\mathrm{b}}$ for no retinopathy compared with some degree of retinopathy

NPDR, non-proliferative diabetic retinopathy

\begin{tabular}{llll}
\hline Characteristic & No progression & Progression & $p$ value \\
\hline Number of patients, $n(\%)$ & $69(86)$ & $11(14)$ & \\
Age (years) & $32.5(5.3)$ & $33.0(5.8)$ & 0.79 \\
Duration of diabetes (years) & $3.3(2.8)$ & $6.7(4.6)$ & 0.03 \\
BMI before pregnancy $\left(\mathrm{kg} / \mathrm{m}^{2}\right)$ & $32.4(7.3)$ & $30.7(4.6)$ & 0.31 \\
HbA 1 (\%) & $6.4(1.0)$ & $7.2(1.2)$ & 0.07 \\
Pre-pregnancy glucose-lowering treatment & & & $0.004^{\mathrm{a}}$ \\
None $(n)$ & 28 & 4 & \\
Oral $(n)$ & 26 & 0 & 0.13 \\
Oral + insulin $(n)$ & 6 & 5 & 0.50 \\
Insulin $(n)$ & 9 & $4(36)$ & 0.18 \\
Smokers, $n(\%)$ & $11(16)$ & $125(18)$ & 0.89 \\
Systolic BP (mmHg) & $121(13)$ & $76(10)$ & 0.36 \\
Diastolic BP (mmHg) & $72(9)$ & $1(9)$ & $0.16^{\mathrm{b}}$ \\
UAE $>30$ mg/24 h, $n(\%)$ & $5(7)$ & $0(0)$ & \\
Antihypertensive treatment before pregnancy, $n(\%)$ & $5(7)$ & 8 & 0.57 \\
Retinopathy, $n$ & & 1 & \\
None & 61 & 0 & \\
Mild NPDR & 6 & $0(0)$ & \\
Moderate NPDR & 2 & & \\
Preproliferative NPDR & 0 & & \\
Proliferative retinopathy & 0 & & \\
Macular oedema, $n(\%)$ & $2(3)$ & & \\
\hline
\end{tabular}


Table 3 Stage of retinopathy for 160 eyes at times indicated in 80 women with type 2 diabetes

Examination at 28 weeks Examination in early pregnancy

\begin{tabular}{lrcccc} 
& No DR & Mild NPDR & Moderate NPDR & Preproliferative NPDR & Proliferative DR \\
\hline No DR & 132 & 4 & 1 & 0 & 0 \\
Mild NPDR & 6 & 4 & 0 & 0 & 0 \\
Moderate NPDR & 7 & 0 & 3 & 0 & 0 \\
Preproliferative NPDR & 0 & 1 & 0 & 0 & 0 \\
Proliferative DR & 0 & 2 & 0 & 0 \\
\hline
\end{tabular}

DR, diabetic retinopathy; NPDR, non-proliferative diabetic retinopathy

initiated antihypertensive treatment, mainly with methyldopa, during pregnancy at a median of 33 weeks (range 19-35).

One woman developing sight-threatening diabetic retinopathy during pregnancy is briefly described in the following. After 5 years of diabetes she had an unplanned pregnancy. On inclusion at 6 weeks, $\mathrm{HbA}_{1 \mathrm{c}}$ was $13.2 \%$, blood pressure 168/97 mmHg, UAE $209 \mathrm{mg} / \mathrm{mmol}$ (albu$\mathrm{min}$ /creatinine ratio) and photoscreening showed mild nonproliferative diabetic retinopathy and visual acuity of 1.0 in both eyes. After initiation of treatment with diet, insulin and methyldopa at 6 weeks, $\mathrm{HbA}_{1 \mathrm{c}}$ decreased to $7.8 \%$ at 10 weeks, to $5.4 \%$ at 22 weeks, remaining at that level until delivery without episodes of hypoglycaemia. Initially compliance with the antihypertensive treatment was suboptimal. At 15 weeks the patient's vision was temporarily impaired to 0.3 on the best eye due to development of CSMO documented with photoscreening and slit lamp biomicroscopy. Proliferations were detected at 19 weeks and panretinal laser therapy was given in the peripheral fundus at 21 and 34 weeks. From 22 weeks onwards the patient was compliant with the antihypertensive treatment and became normotensive on methyldopa, labetalol and thiazid. At 35 weeks she developed preeclampsia and was delivered by Caesarean section. At 1 week postpartum visual acuity was 1.0 and 0.7 , respectively. Due to progression of the macular lesions, the patient received laser treatment in the macular region of the left eye 1 month after pregnancy. The proliferations and macular oedema on the right eye have now fully regressed.

Two women with mild to moderate macular oedema and normal visual acuity in early pregnancy experienced regression of diabetic retinopathy during pregnancy; their $\mathrm{HbA}_{1 \mathrm{c}}$ levels declined from $6.7 \%$ to $6.4 \%$ and from $7.7 \%$ to $5.0 \%$, respectively.

The prevalence of preeclampsia and preterm delivery was similar in women with and without progression (Table 5). One woman without progression experienced a stillbirth at 39 weeks.

Diabetes duration, the occurrence of diabetic retinopathy and the pregnancy outcome in the 28 excluded women with less than two eye examinations during pregnancy were comparable with the included women (data not given).

\section{Discussion}

In an unselected population of pregnant women with type 2 diabetes, diabetic retinopathy was present in early pregnancy; retinopathy progressed during pregnancy in 14\% of these women. Progression of diabetic retinopathy was mainly mild, but sight-threatening diabetic retinopathy was seen in one woman with poor pre-pregnancy glycaemic control, low
Table 4 Glycaemic control and blood pressure during pregnancy in women with progression of retinopathy $(n=11)$ compared with women without progression of diabetic retinopathy $(n=69)$

Data are given as mean (SD); there were no significant differences between progression and no progression at any timepoint

${ }^{\mathrm{a}} n=52-55$

DR, diabetic retinopathy

\begin{tabular}{lccccc}
\hline DR per clinical variable & \multicolumn{4}{l}{ Mean gestational weeks } \\
\cline { 2 - 5 } & 10 weeks & 14 weeks & 22 weeks & 28 weeks & 34 weeks \\
\hline $\mathrm{HbA}_{1 \mathrm{c}}(\%)$ & & & & \\
No progression & $6.5(1.1)^{\mathrm{a}}$ & $6.0(0.7)$ & $5.5(0.5)$ & $5.6(0.6)$ & $5.7(0.6)$ \\
Progression & $7.2(1.2)$ & $6.3(0.7)$ & $5.4(0.5)$ & $5.5(0.5)$ & $5.7(0.6)$ \\
Systolic BP (mmHg) & & & & & \\
No progression & $121(11)^{\mathrm{a}}$ & $124(13)$ & $120(12)$ & $122(14)$ & $125(16)$ \\
Progression & $123(17)$ & $125(15)$ & $124(15)$ & $122(10)$ & $123(18)$ \\
Diastolic BP (mmHg) & & & & & $72(9)$ \\
$\quad$ No progression & $71(9)^{\mathrm{a}}$ & $74(9)$ & $71(8)$ & $72(9)$ \\
Progression & $77(10)$ & $75(9)$ & $75(7)$ & $73(7)$ & $75(7)$ \\
\hline
\end{tabular}


Table 5 Pregnancy-induced clinical characteristics in relation to progression of diabetic retinopathy in 80 women with type 2 diabetes

Values are mean (SD) unless otherwise stated

${ }^{\mathrm{a}}$ Insulin treatment compared with no insulin treatment at 10 weeks; ${ }^{\mathrm{b}}$ no antihypertensive treatment at all compared with antihypertensive treatment

\begin{tabular}{llll}
\hline Variables & No progression & Progression & $p$ value \\
\hline Pregnancy glucose-lowering treatment $(n)$ & & & $0.04^{\mathrm{a}}$ \\
$\quad$ Diet alone & 6 & 0 & \\
Diet + insulin after 10 weeks & 14 & 0 \\
Insulin at 10 weeks & 34 & 4 & \\
Insulin before pregnancy & 15 & 7 & 0.30 \\
Insulin dose (IU/kg) at 34 weeks & $1.14(0.83)$ & $1.41(0.71)$ & 0.95 \\
Weight gain from pre-pregnancy to 34 weeks $(\mathrm{kg})$ & $11.9(6.1)$ & $12.0(6.7)$ & 0.63 \\
Antihypertensive treatment $(n)$ & $(59,5,5)$ & $10,1,0)$ & \\
None & 59 & 1 & \\
Added during pregnancy & 5 & 0 & 0.68 \\
Since pre-pregnancy & 5 & $3(27)$ & 0.44 \\
Preeclampsia, $n(\%)$ & $4(6)$ & $12(17)$ & \\
Premature delivery, $n(\%)$ & & & \\
\hline
\end{tabular}

compliance to treatment and hypertension in the first part of pregnancy.

One strength of the study is that the population was an unselected cohort and that the retinopathy status of women excluded was taken into account. Another strength is that grading of the fundus photography was performed by the same ophthalmologist with access to both sets of photographs using standardised assessment of diabetic retinopathy including macular oedema. In addition measures of glycaemic control as well as blood pressure were carefully documented throughout the pregnancies.

A limitation of this study is that only $73 \%$ of the women had the available ophthalmological examinations during pregnancy and that postpartum examinations were only performed in one woman with sight-threatening diabetic retinopathy. Development of diabetic retinopathy in late pregnancy and postpartum may be important [12], but a recent study suggests that this is not a major issue [13]. The relatively small sample size makes it impossible to analyse the independent roles of pre-pregnancy and intra-pregnancy changes in $\mathrm{HbA}_{1 \mathrm{c}}$, blood pressure and smoking. An agematched control group of non-pregnant women with similar duration of type 2 diabetes and serial photoscreenings performed in a similar way was not possible to obtain. The role of pregnancy for the progression observed can therefore not be evaluated in this study. The number of pregnant and non-pregnant women included in a comparative study needs to be very high in order to evaluate this question properly. The examinations of the photoscreening pictures were performed by our experienced ophthalmologist, which is a good reflection of the routine clinical situation. An examination of the pictures by two independent observers blinded to patient characteristics could have improved the accuracy of picture grading.

Presence of macular oedema in early pregnancy in women with type 1 diabetes has been associated with sight-threatening deterioration of retinopathy during pregnancy [14]. It is therefore surprising that the two women with macular oedema in early pregnancy in the current study regressed during pregnancy. However, the numbers are too small to make any firm conclusions.

In early pregnancy the majority of women had no or mild retinal changes, which is comparable to previous reports on women with type 1 diabetes with similar duration of diabetes $[14,15]$. The risk of progression of diabetic retinopathy in women with type 2 diabetes in the current study seems to be lower than in women with type 1 diabetes [15]. However, since sight-threatening deterioration of diabetic retinopathy occurred in one pregnant women with type 2 diabetes in the current study and in a woman with type 2 diabetes described by us in a previous cohort [4], as well as in a Japanese study of women with type 2 diabetes [2], the risk of progression cannot be ignored.

The woman with pregnancy-induced sight-threatening diabetic retinopathy had extremely poor glycaemic control and untreated hypertension related to presence of diabetic nephropathy in early pregnancy. This type of patient does not occur frequently, but all centres probably have an incompliant patient like this from time to time. This single case of progression to proliferative retinopathy suggests that rapid improvement of glycaemic control and blood pressure could be responsible for progression of retinopathy during pregnancy in patients with poorly controlled glycaemia before pregnancy.

In the group as a whole no statistically significant associations between progression of diabetic retinopathy or blood pressure and baseline levels of $\mathrm{HbA}_{1 \mathrm{c}}$ were found. This might be due to the relatively small material giving a high risk of type 2 error; it could also be due to the fact that the included women had sufficiently tight glycaemic control in early pregnancy to prevent this association from becoming evident. In women with type 1 diabetes an association of 
high levels of $\mathrm{HbA}_{1 \mathrm{c}}$ and blood pressure with pregnancyinduced progression of retinopathy is well described [16, 17], but in a study with sufficiently strict glycaemic control this was not found [18]. Optimising glycaemic control before pregnancy in type 1 diabetes is associated with a tendency towards less progression compared with non-optimising of glycaemic control until pregnancy is confirmed [19]. In the current study, the decrease in $\mathrm{HbA}_{1 \mathrm{c}}$ from baseline to 34 weeks was significantly larger in women with progression of diabetic retinopathy. This underlines the importance of lowering $\mathrm{HbA}_{1 \mathrm{c}}$ before pregnancy. Strict metabolic control is of uppermost importance for the outcome of pregnancy, since the occurrence of preeclampsia, preterm delivery and stillbirth is related to poor metabolic control [20-23]. The prevalence of perinatal mortality tended to be lower in this cohort than in previous observations from our centre [5]. The low incidence of severe progression of retinopathy during pregnancy observed by us in women reaching an average $\mathrm{HbA}_{1 \mathrm{c}}$ of $5.6 \%$ from pregnancy week 20 is reassuring for continuing a strategy of strict metabolic control. It is well established that strict metabolic control prevents progression of retinopathy in type 1 [24] and type 2 diabetes [25]. However, it has been reported that a minority of diabetic patients with type 1 diabetes can develop significant sight-threatening deterioration of diabetic retinopathy in association with initiation of strict glycaemic control in the pregnant [26] and in the non-pregnant state [27]. In the long run, it seems that even these patients with early worsening of retinopathy benefit from strict metabolic control [28]. It remains a matter for speculation whether less strict metabolic control during pregnancy should be aimed for in selected pregnant women with poor metabolic control and a high risk of severe progression of retinopathy.

Pregnancy-induced progression of retinopathy was associated with indicators of more severe type 2 diabetes as reflected by increased diabetes duration and the need for insulin treatment prior to pregnancy. This relation to diabetes duration is also seen in type 1 diabetes [18, 29, 30]. Taking into account that the known duration of diabetes was shorter in this population of pregnant women with type 2 diabetes and comparing the retinopathy data of a population of pregnant women with type 1 diabetes investigated by the same method in the same centre [14], we estimate that the risk of progression of diabetic retinopathy is comparable in type 1 and type 2 diabetes.

The pathophysiological factors involved in pregnancyinduced progression of retinopathy [1] are probably similar in type 1 and type 2 diabetes.

Despite a low risk of progression of diabetic retinopathy in pregnant women with type 2 diabetes, development of sight-threatening diabetic retinopathy did occur. Screening for diabetic retinopathy in pregnancy among women with type 2 diabetes should therefore follow the same recom- mendations as those applying for women with type 1 diabetes.

Acknowledgements We would like to acknowledge project nurses C. Barfred, E. Stage and A. Bæk, laboratory technician K. M. Larsen and secretary S. Graversen for kind assistance with the data collection process. We would also like to acknowledge the Ophthalmological Clinic at Rigshospitalet.

Duality of interest E. R. Mathiesen has received fees for speaking from Aventis and Novo Nordisk, and funds for research and fees for consulting from Novo Nordisk. P. Damm has received funds for research, and fees for consulting and speaking from Novo Nordisk. For M. Vestgaard, L. Ringholm, C. S. Laugesen. and K. L. Rasmussen there is no duality interest associated with this manuscript.

\section{References}

1. Kaaja R, Loukovaara S (2007) Progression of retinopathy in type 1 diabetic women during pregnancy. Curr Diabetes Rev 3:85-93

2. Omori Y, Minei S, Testuo T, Nemoto K, Shimizu M, Sanaka M (1994) Current status of pregnancy in diabetic women. A comparison of pregnancy in IDDM and NIDDM mothers. Diabetes Res Clin Pract 24(Suppl):S273-S278

3. Confidential Enquiry into Maternal and Child Health (2005) Pregnancy in women with type 1 and type 2 diabetes in 2002-03, England, Wales and Northern Ireland. Available from www. cmace.org.uk/Publications/Diabetes\%20Report/content.htm, accessed 15 April 2009

4. Rasmussen KL, Laugesen CS, Datta N, Damm P, Mathiesen ER (2008) Diabetic retinopathy during pregnancy. Ugeskr Laeger 170:4117-4121 (Article in Danish)

5. Clausen TD, Mathiesen E, Ekbom P, Hellmuth E, MandrupPoulsen T, Damm P (2005) Poor pregnancy outcome in women with type 2 diabetes. Diabetes Care 28:323-328

6. Feig DS, Palda VA (2002) Type 2 diabetes in pregnancy (a growing concern). Lancet 359:1690-1692

7. Wilkinson CP, Ferris FL, Klein RE et al (2003) Proposed international clinical diabetic retinopathy and diabetic macular edema disease severity scales. Ophthalmology 110:1677-1682

8. Early Treatment Diabetic Retinopathy Study Research Group (1991) Early photocoagulation for diabetic retinopathy. Ophthalmology 98:766-785

9. Nielsen LR, Ekbom P, Damm P et al (2004) HbAlc levels are significantly lower in early and late pregnancy. Diabetes Care 27:1200-1201

10. Nielsen LR, Damm P, Mathiesen ER (2009) Improved pregnancy outcome in type 1 diabetic women with microalbuminuria or diabetic nephropathy: effect of intensified antihypertensive therapy? Diabetes Care 32:38-44

11. Feldt-Rasmussen B, Dinesen B, Deckert M (1985) Enzyme immunoassay: an improved determination of urinary albumin in diabetics with incipient nephropathy. Scand J Clin Lab Invest 45:539-554

12. Hellstedt T, Kaaja R, Teramo K, Immonen I (1997) The effect of pregnancy on mild diabetic retinopathy. Graefes Arch Clin Exp Ophthalmol 235:437-441

13. Arun CS, Taylor R (2008) Influence of pregnancy on long-term progression of retinopathy in patients with type 1 diabetes. Diabetologia 51:1041-1045

14. Vestgaard M, Nielsen LR, Laugesen CS, Damm P, Mathiesen ER (2009) Pregnancy-induced sight threatening diabetic retinopathy in women with type 1 diabetes. Diabetologia:A09 (Abstract) 
15. Temple RC, Aldridge VA, Sampson MJ, Greenwood RH, Hayburn PJ, Glenn A (2001) Impact of pregnancy on the progression of diabetic retinopathy in type 1 diabetes. Diabet Med 18:573-577

16. Chew EY, Mills JL, Metzger BE et al (1995) Metabolic control and progression of retinopathy. Diabetes Care 18:631-637

17. Rosenn B, Miodovnik M, Kranias G et al (1992) Progression of diabetic retinopathy in pregnancy: association with hypertension in pregnancy. Am J Obstet Gynecol 166:1214-1218

18. Lauszus F, Klebe JG, Bek T (2000) Diabetic retinopathy in pregnancy during tight metabolic control. Acta Obstet Gynecol Scand 79:367-370

19. The Diabetes Control and Complications Trial research group (2000) Effect of pregnancy on microvascular complications in the Diabetes Control and Complications Trial. Diabetes care 23:10841091

20. Mathiesen ER, Vaz JA (2008) Insulin treatment in diabetic pregnancy. Diabetes Metab Res Rev 24:S3-S20

21. Ekbom P, Damm P, Feldt-Rasmussen B, Feldt-Rasmussen U, Mathiesen ER (2008) Elevated haemoglobin A1c in the third trimester is related to preterm delivery in type 1 diabetes. J Diabetes Complications 22:297-302

22. Lauenborg J, Mathiesen ER, Ovesen P, Westergaard JG, Ekbom P, Damm P (2003) Audit on stillbirths in women with pregestational type 1 diabetes mellitus. Diabetes Care 26:1385-1389

23. Hiilesmaa V, Suhonen L, Teramo K (2000) Glycaemic control is associated with pre-eclampsia but not with pregnancy-induced hypertension in women with type 1 diabetes mellitus. Diabetologia 43:1534-1539

24. Diabetes Control and Complications Trial research group (1993) The effect of intensive treatment of diabetes on the development and progression of long-term complications of insulin dependent diabetes mellitus. N Engl J Med 329:977-986

25. UK Prospective Diabetes Study (UKPDS) group (1998) Intensive blood-glucose control with sulphonylureas or insulin compared with conventional treatment and risk of complications in patients with type 2 diabetes (UKPDS 33). Lancet 352:837-853

26. Phelps RL, Sakol P, Metzger BE, Jampol LM, Freinkel N (1986) Changes in diabetic retinopathy during pregnancy. Correlations with regulation of hyperglycemia. Arch Ophtalmol 104:1806 1810

27. Lauritzen T, Frost-Larsen K, Larsen HW, Deckert T, Steno Study Group (1983) Effects of one year of near normal blood glucose levels on retinopathy in insulin dependent diabetics. Lancet 1:200-204

28. Diabetes Control and Complications Trial research group (1998) Early worsening of diabetic retinopathy in the Diabetes Control and Complications Trial. Arch Ophthalmol 116:874-886

29. Vérier-Mine O, Chaturvedi N, Webb D, Fuller JH (2005) Is pregnancy a risk factor for microvascular complications? The EURODIAB prospective complications study. Diabet Med 22:1503-1509

30. Axer-Siegel R, Hod M, Fink-Cohen S et al (1996) Diabetic retinopathy during pregnancy. Ophthalmology 103:1815-1819 